\author{
Military Technical \\ College \\ Kobry El-Kobba \\ Cairo, Egypt
}

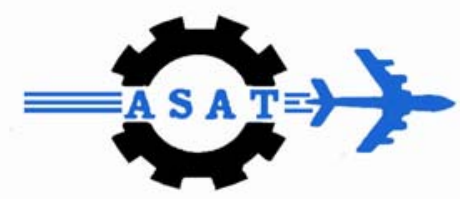

12-th International

Conference on

Aerospace Sciences \&

Aviation Technology

\title{
SMALL SIGNAL GAIN DEGRADATION AND ITS CORRECTION BY FEEDFORWARD LINEARIZATION
}

\author{
AHMAD SELEEM MAHMOUD ${ }^{1}$, A. H. ASSISI ${ }^{1}$ and MOSTAFA M. IBRAHIM ${ }^{1}$
}

\begin{abstract}
In this paper we propose a new measure of amplifier nonlinearity; namely, the small signal gain degradation due to large signal capture of the amplifier power. This new criterion for linearity evaluation is specially important in repeaters where received signals are not a priory known. A $5^{\text {th }}$ order mathematical model is used to characterize this phenomenon. After a thorough comparison between different linearization techniques, we select the classical feed-forward linearization. We adapt the FF linearization circuit to the total input signal power at different conditions and simulate the small signal gain against a large input signal gain in each case. An interesting result was that the feed-forward linearization circuit adapted to the total input power, completely compensates the small signal gain degradation. Although the results are frequency dependent, we can find some average settings to correct the system performance within a certain frequency band. We propose the use of a general power detector and a set of look-up tables for system adaptation.
\end{abstract}

\section{KEY WORDS}

Small signal gain degradation, feed-forward, adaptive feed-forward, linearization, AM-AM conversion, AM-PM conversion, Harmonic Distortion, Inter-modulation Distortion, Gain Compression, phase Distortion, and adjacent Channel Interference.

\section{NOMENCLATURE}

$\begin{array}{ll}\text { ADS } & \text { Advanced design system } \\ \text { FF } & \text { Feed-Forward } \\ \text { IBIP } & \text { In-Band Inter-modulation Products } \\ \text { IMD }_{3} & \text { Third-order Inter-modulation Distortion } \\ \mathbf{P}_{1 \mathrm{~dB}} & 1 \mathrm{~dB} \text { compression point } \\ \mathbf{P}_{\mathrm{IP3}} & \text { Third order Intercept point }\end{array}$

\footnotetext{
${ }^{1}$ Egyptian Armed Forces
} 


\section{INTRODUCTION}

RF and microwave power amplifiers are used in a wide variety of applications. All wireless transmitters contain RF amplifiers which are nonlinear to some degree. Nonlinearities cause imperfect reproduction of the amplified signal if the signal to be amplified has a non-constant envelope, such as for linear modulation schemes or for a multicarrier signals [1]. The most important nonlinear effects in a nonlinear power amplifier are Harmonic Distortion, Inter-modulation Distortion IMD, Gain Compression, phase Distortion [phase deviation as a function of amplitude or power level], and Adjacent Channel Interference.

When the envelope of the signal is clipped or phase rotated, the resulting IMD (Spectral Re-growth) causes out-of-band components in adjacent channels, thus causing interference to other users of the system. For this reason the IMD of digitally modulated signals are often specified as adjacent channel power ratio (ACPR) [2]. AM-AM conversion is the modification in the fundamental signal gain as the input amplitude is increased. AM-PM conversion is a phase change in the fundamental signal introduced by AM-PM dependence on signal amplitude

These nonlinear effects can be characterized by different criteria. The $1 \mathrm{~dB}$ compression point $\mathrm{P}_{1 \mathrm{~dB}}$ is the output power level, at which the gain has dropped by 1 $\mathrm{dB}$ compared to the linear gain [3]. The third order Intercept point $\mathrm{P}_{\mathrm{IP} 3}$ is the output power level corresponding to the intersection of the extrapolation of the fundamental, and the third order IMD [2].

In a signal repeater, such as a communication repeater or a repeater jammer, where the received signals are not a priory known, nonlinearity has another important effect that degrades the repeater performance. The characterization and compensation of this additional effect is the subject of this paper.

\section{DESCRIPTION OF THE PROBLEM}

\section{Mathematical Formulation}

Consider a nonlinear power amplifier modeled with an $\mathrm{n}^{\text {th }}$ order power series transfer characteristics

$$
V_{\text {out }}(t)=\sum_{i=1}^{n} a_{i} V_{\text {in }}^{i}(t)
$$

Consider a two-tone signal $x(t)$ applied to this amplifier

$$
x(t)=A_{1} \cos \left(\omega_{1} t\right)+A_{2} \cos \left(\omega_{2} t\right), \omega_{1}<\omega_{2}
$$

Only odd-order terms are responsible for the In-Band Inter-modulation Products (IBIP), while the even terms, are responsible for the out-of-band distortion products [3]. Considering terms up to the fifth-order and neglect higher-order terms due to their smaller weights in the output spectrum and simplify our analysis with acceptable 
accuracy. From (1) and (2), it is easy to derive an expression for the output spectrum of the amplifier. If we divide the coefficient of $\cos \left(\omega_{1} t\right)$ by $A_{1}$ and that of $\cos \left(\omega_{2} t\right)$ by $A_{2}$ we get the voltage gain for each of the two input signals:

$$
\begin{aligned}
& g_{1}=a_{1} A_{1}+\frac{3 a_{3} A_{1}^{2}}{4}+\frac{3 a_{3} A_{2}^{2}}{2}+\frac{5 a_{5} A_{1}^{4}}{8}+\frac{15 a_{5} A_{2}^{4}}{8}+\frac{30 a_{5} A_{1}^{2} A_{2}^{2}}{8} \\
& g_{2}=a_{1} A_{2}+\frac{3 a_{3} A_{2}^{2}}{4}+\frac{3 a_{3} A_{1}^{2}}{2}+\frac{5 a_{5} A_{2}^{4}}{8}+\frac{15 a_{5} A_{1}^{4}}{8}+\frac{30 a_{5} A_{1}^{2} A_{2}^{2}}{8}
\end{aligned}
$$

Equal signals will have equal gain and equal pairs of spurious outputs symmetrically distributed around the two input frequencies.

If the amplitude of the first tone is much smaller than that of the second $\left(A_{1}<<A_{2}\right)$, while the second signal is strong enough to drive the amplifier into compression, the in-band output of the PA can be approximated to

$$
\begin{aligned}
V_{\text {out }}(t) \cong\left(a_{1} A_{1}+\frac{3 a_{3} A_{1} A_{2}^{2}}{2}+\frac{15 a_{5} A_{1} A_{2}^{4}}{8}\right) \cos \left(\omega_{1} t\right) \\
+\left(a_{1} A_{2}+\frac{3 a_{3} A_{2}^{3}}{2}+\frac{5 a_{5} A_{2}^{5}}{4}\right) \cos \left(\omega_{2} t\right) \\
+\left(\frac{3 a_{3} A_{1} A_{2}^{2}}{4}+\frac{5 a_{5} A_{1} A_{2}^{4}}{4}\right) \cos \left(2 \omega_{2}-\omega_{1}\right) t
\end{aligned}
$$

And the two gains become:

$$
\begin{aligned}
& g_{1} \cong\left(a_{1}+\frac{3 a_{3} A_{2}^{2}}{2}+\frac{15 a_{5} A_{2}^{4}}{8}\right) \\
& g_{2} \cong\left(a_{1}+\frac{3 a_{3} A_{2}^{2}}{4}+\frac{5 a_{5} A_{2}^{4}}{8}\right)
\end{aligned}
$$

We can notice the following:

- The coefficient of $\cos \left[\left(2 \omega_{1}-\omega_{2}\right) t\right]$ contains powers of $A_{1}$ higher than one and can be neglected; which means that this inter-modulation frequency component will have a very small output power.

- All fifth (and higher order) inter-modulation products contain higher powers of $A_{1}$ and can be neglected.

- The gain of the power amplifier will not equally distribute on the two signals; i.e. $g_{2}$ is not equal to $g_{1}$.

At a first glance, $g_{2}$ seems smaller than $g_{1}$, but if we consider that for a saturated power amplifier, usually $a_{3}$ and $a_{5}$ are negative, or at least one of them. We understand how the power amplifier gain is captured by the strong signal.

This degradation of small signal gain due to the presence of large signal is the proposed measure of power amplifier nonlinearity. We can call this phenomenon small signal gain degradation, or simply Gain Capture. It degrades the performance 
of power amplifiers working at or near saturation, such as communication power amplifiers, where efficiency requirements force designers to bias the PA in classes $C$, $D, E$ and even $F$. It degrades, also, wide-band saturated power amplifiers in jamming systems. Moreover, it affects wide-open receiving amplifiers in EW systems, where neither signal frequencies nor their levels are a-priori known. In such receivers, gain degradation can define the upper limit of dynamic range.

\section{Verification by ADS CAD simulations}

To verify the gain capture problem, using the nonlinear model of the Agilent Va-hpMSA-0500 power amplifier at 1, 1.5, and $2 \mathrm{GHz}$, and a two-tone input signal with different tones power (weak signal and strong signal) at $2[\mathrm{kHz}]$ frequency spacing. The total input power is then:

$$
P_{\text {total }}=10 * \log \left(\frac{A_{1}^{2}+A_{2}^{2}}{2 R}\right)+30 \quad[\mathrm{dBm}]
$$

Where $\mathrm{A} 1$ and $\mathrm{A} 2$ are the input signal amplitudes [v]

$R$ is the system characteristic impedance $[\Omega]$

Sweeping the strong signal power from $-7 \mathrm{dBm}$ to $+5 \mathrm{dBm}$, while keeping the weak signal power at $-20 \mathrm{dBm}$, and measure the signals gain in $\mathrm{dB}$ at each measurement. Simulation ensure that the weak signal gain is decreased rapidly as the level of the higher tone is increased, while the higher tone captured the gain of the amplifier receiving most of its power.

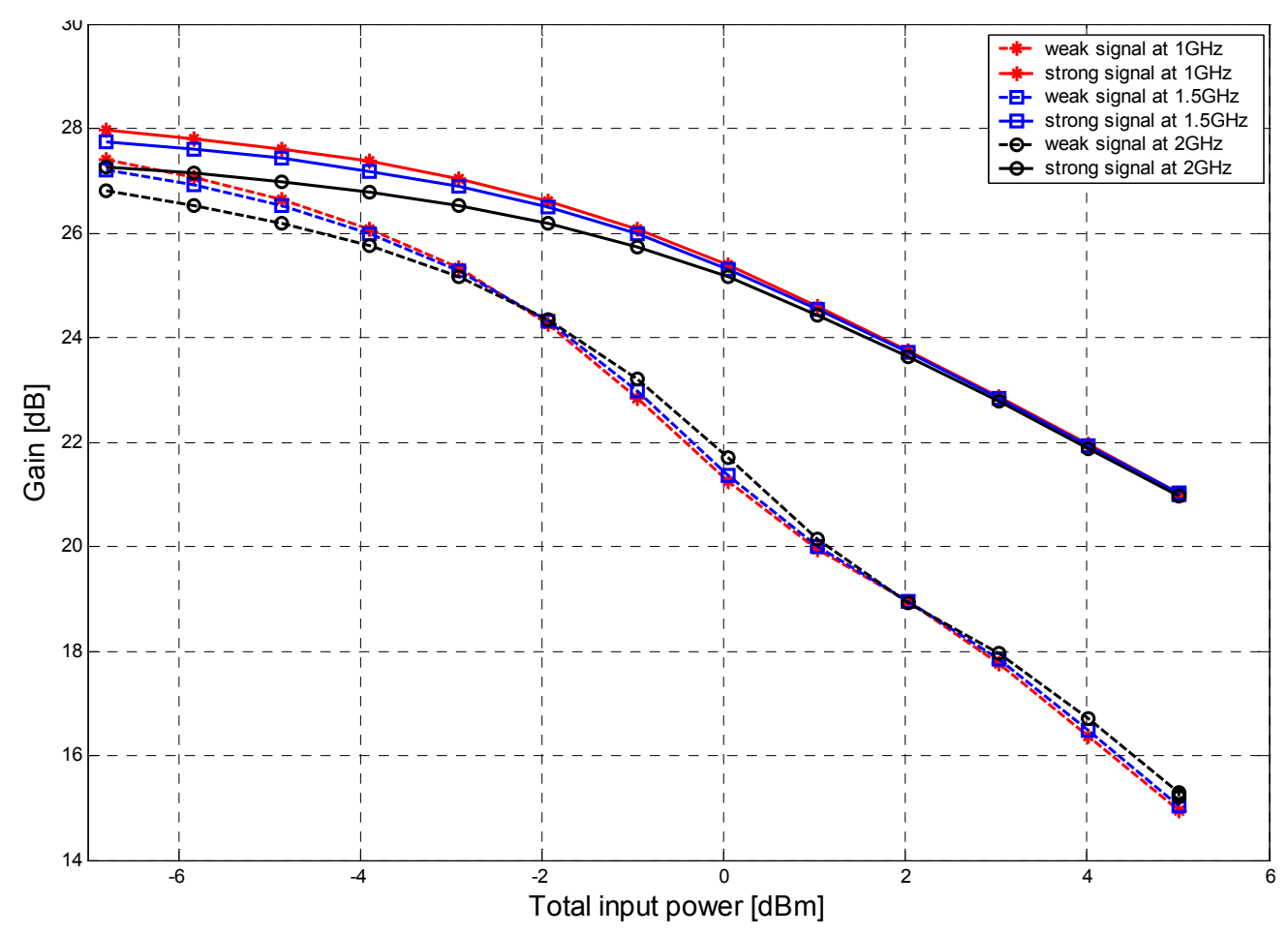

Figure 1: Gain Capture of the PA at 1, 1.5 and $2 \mathrm{GHz}$ 
As the input power level increase the gain difference between the large and the small signal increases and the gain difference for each signal at different frequencies decrease. As shown in figure 1.

\section{SOLUTION OF THE GAIN CAPTURE PROBLEM}

After describing the problem, and since the problem reason is amplifier nonlinearity, the solution is to linearize the power amplifier characteristics using external circuit.

\section{1- Survey of different linearization techniques}

Several linearization techniques exist. They were discussed in details in [2], [3] and [4]. Only the most common categories are briefly mentioned here.

Feedback techniques can suppress distortion as long as the feedback loop has sufficient incremental gain [1]. To increase the loop gain, base-band error amplifiers in Cartesian or polar form are commonly used. The necessary up and down conversions inside the loop increase noise sources and loop delays, limiting the stable bandwidth of the linearizer typically to below $100 \mathrm{kHz}$ [3]. We cannot use feedback techniques to correct the gain capture problem for signal durations shorter than the feedback delay.

Predistortion based on expanding the signal before the power amplifier, so that the predistorter-amplifier pair appears as a linear circuit [3]. In principle, predistortion is a very power efficient and wideband linearization method, although it typically needs a slow feedback to adapt the predistortion function. A simple RF predistorter may consist of just a couple of biased diodes [1], or the predistortion signal can be generated in the digital baseband using adapted lookup tables. Predistortion techniques need a-priori knowledge of the signal spectrum and cannot be used in applications such as EW receivers or jammers.

Feedforward linearization (FF), is the most effective and popular technique for improving the linearity of power amplifiers, due to its potential for excellent distortion suppression [3], it is commonly used in wideband amplifiers. The distortion generated in the main amplifier is extracted by canceling the linear signal from the output of the main amplifier. This distortion signal is amplified by an auxiliary amplifier and finally subtracted from the PA output. As this arrangement does not contain a feedback loop, it has no stability limitations, but still the bandwidth of the power combiners and phase shifters limits the cancellation bandwidth. Feedforward linearization has advantages in speed, stability due to its open-loop nature. Another advantage is the operating bandwidth; generally, it is used with very wide bandwidth multi-carrier applications [6]. Although a classical method, it seems to be the most suitable technique for our problem. We apply Feedforward linearization with some sort of realtime adaptation to solve the gain capture problem. A short comparison between common linearization techniques is present in Table 1. 
Table 1: Comparison between different linearization techniques

\begin{tabular}{|c|c|c|c|}
\hline & Feedback & Feedforward & Predistortion \\
\hline Bandwidth & Narrow & Wide & Wide \\
\hline Efficiency & Low & medium & Intermediate \\
\hline Complexity & Low & High & $\begin{array}{c}\text { Can overcome stability } \\
\text { problems }\end{array}$ \\
\hline Stability & $\begin{array}{c}\text { There is stability } \\
\text { problems }\end{array}$ & Unconditionally stable & Intermediate \\
\hline Cost & Low & High & \\
\hline
\end{tabular}

\section{2- Simulation of Feedforward Linearization Technique}

The proposed linearized amplifier system, shown in Figure 2, consists of a two-stage power amplifier, the first stage is a $20 \mathrm{~dB}$ linear amplifier $\left(\mathrm{A}_{1}\right)$, and the second stage is a nonlinear power amplifier $\left(\mathrm{A}_{2}\right)$ with a typical $8.5 \mathrm{~dB}$ gain, a feedforward linearization circuit, and a digital control circuit. The linearizer consists of two loops, the first is the signal cancellation loop, and the second is the error cancellation loop. In the signal cancellation loop, a portion of the power amplifier input is fed, through a10-dB directional coupler, to the reference path, where it is delayed to compensate for the PA group delay and then attenuated if necessary, to be subtracted from an attenuated sample of the PA output to suppress the original signal and get a pure spurious signal. This spurious signal is amplified and subtracted from the PA output to get a spur-free output.

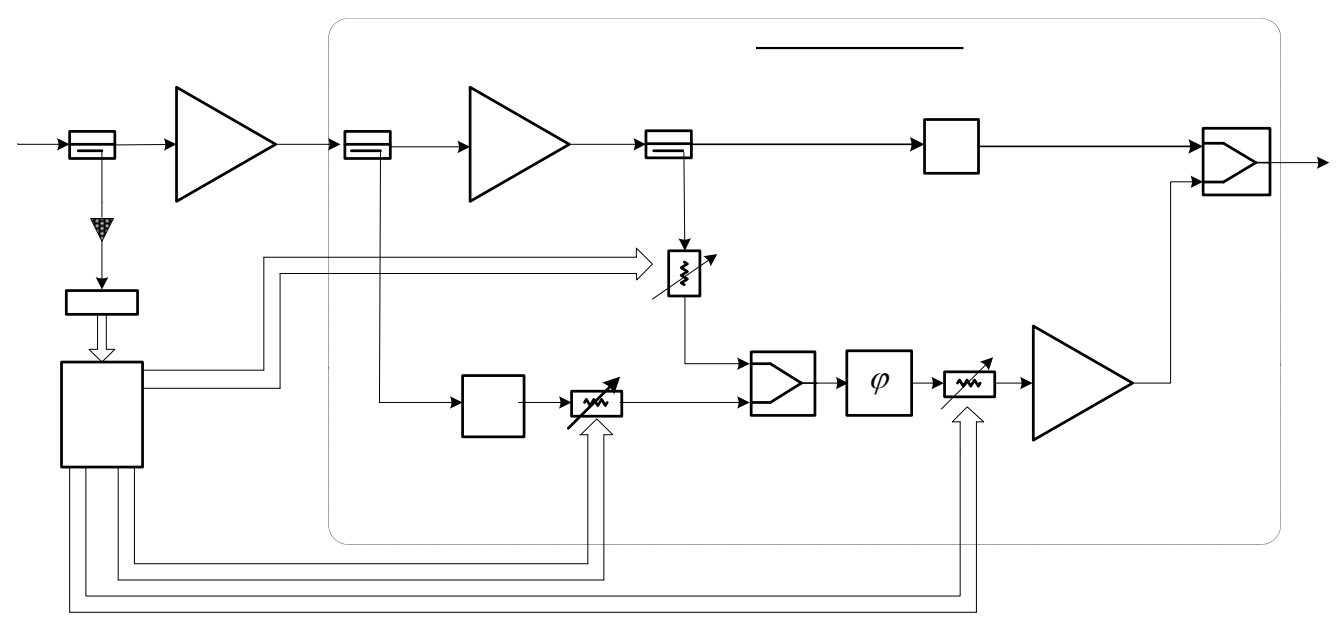

Figure 2: Adaptive Feedforward system block diagram

The function of the digital control circuit is to adjust the values of the set of attenuators for perfect cancellation performance at each power combiner, which in this case will work as a subtractor. For every different total input power level, each attenuator will require a specific value. We run a two-tone ADS simulation, with equal two-tone input to calculate the accurate attenuation values at each different level of the total input power. The attenuators were set in each case to obtain the maximum possible IM3 reduction. These attenuator settings were stored in a Look-up table (LUT). The power sensor at the input coupled port of the system, together with the 
$A / D$ converter, are responsible for choosing the required attenuation setting at each new value of the total input power. Again, we run the simulation at $1,1.5$ and $2 \mathrm{GHz}$ to cover an octave frequency band, and the overall results were stored in three lookup tables, one for each frequency

\section{3- Adaptive FF linearization as a gain capture correction technique}

After adjusting the attenuation values for maximum IMD cancellation at a certain value of total input power, two different signals with the same power sum were applied to the system input, such that

$$
10 \log \left(P_{S}+P_{L}\right)=10 \cdot \log P_{T T}+3 d B
$$

Where $P_{S}, P_{L}$ and $P_{T T}$ are the small, large and two-equal tone signals, respectively.

Simulation results showed that the linearization system was capable of completely correcting the gain capture effect for a certain total input power when the attenuators are set to cancel the two-equal tone spurs for the same total input power. At the output, both small and large signals get equal gains. Figures 2, 3 and 4 show the simulation results at $1,1.5$, and $2 \mathrm{GHz}$ respectively.

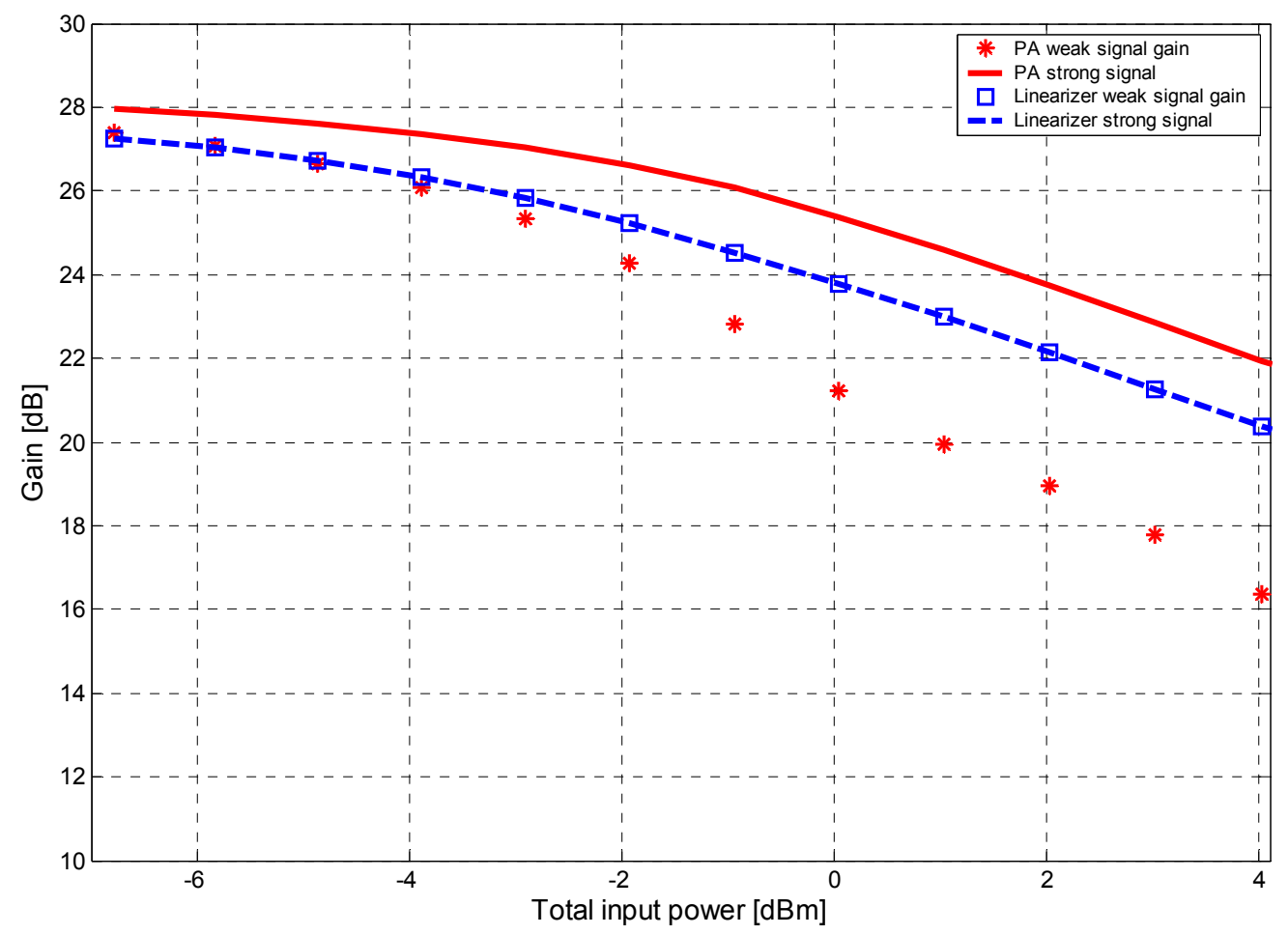

Figure 3: small and large signal gains before and after linearization at $1 \mathrm{GHz}$ 


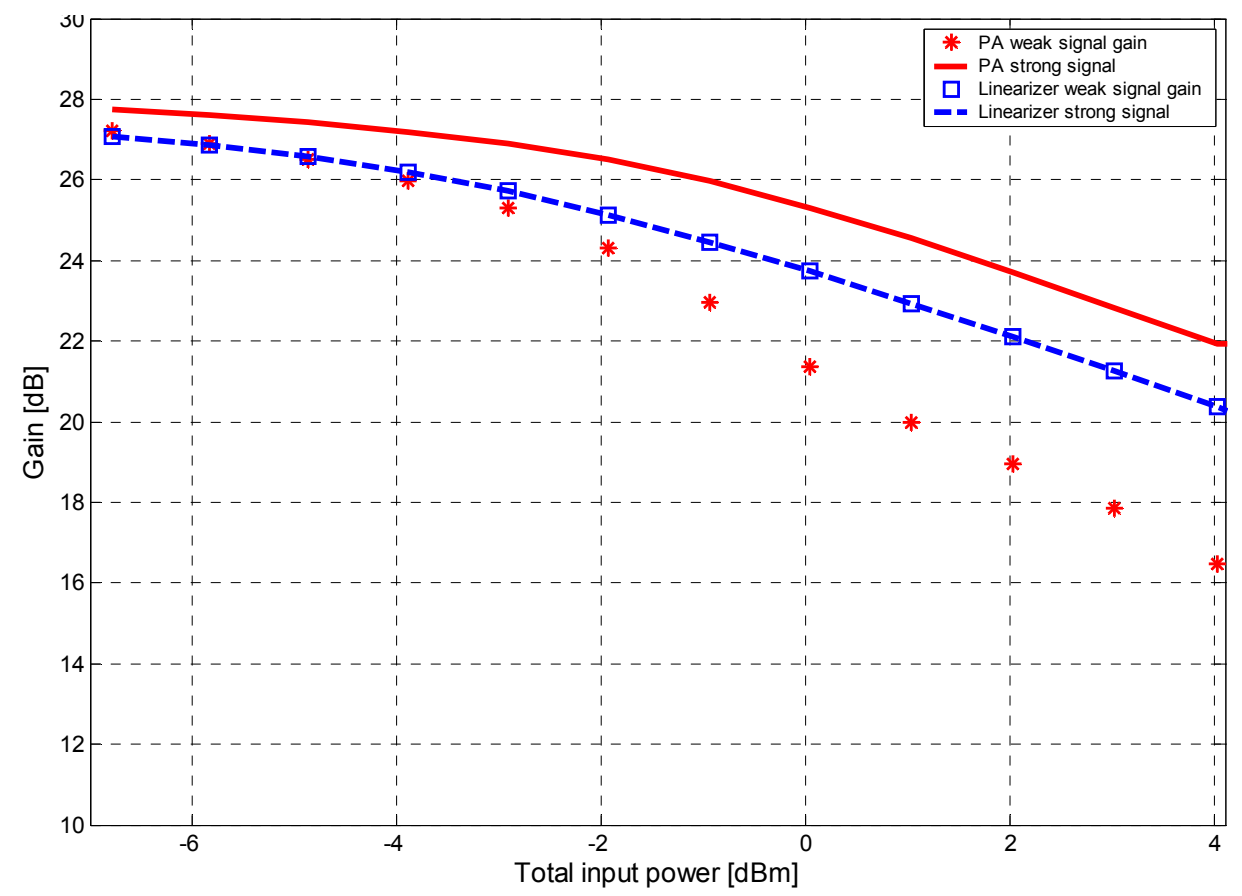

Figure 4: small and large signal gains before and after linearization at $1.5 \mathrm{GHz}$

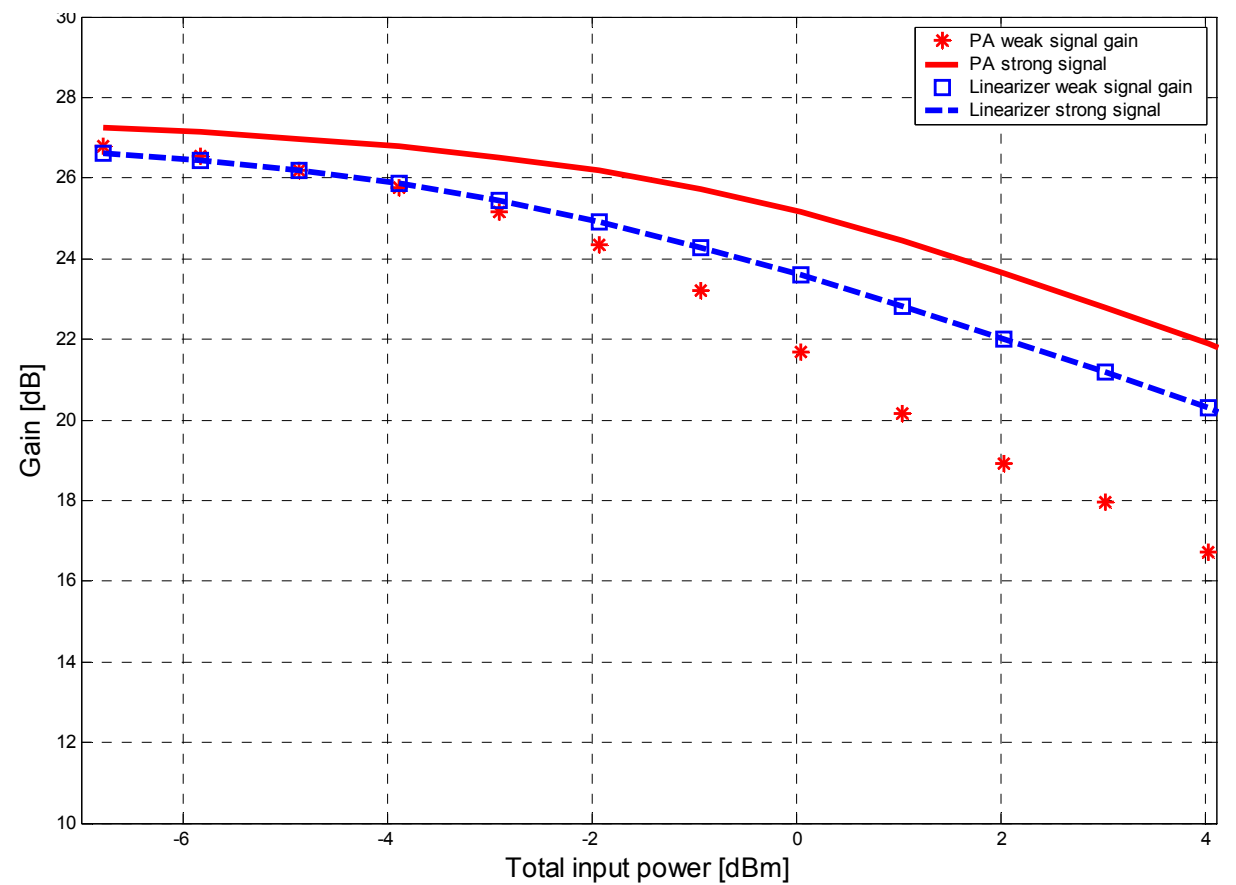

Figure 5: small and large signal gains before and after linearization at $2 \mathrm{GHz}$

\section{4- System Adaptation on an octave frequency band.}

Using a look-up table for each frequency requires a frequency measurement device to determine the signal frequency and select the suitable table. This is a complex design requirement. Moreover, in case of simultaneous input signals with different frequencies it becomes impossible. We tried to solve this problem and simplify the system adaptation by using only one look-up table with average values of attenuator setting covering the total frequency band. 
Simulation results using the average attenuation settings, showed a maximum of 0.4 $\mathrm{dB}$ deviation in the system performance between using accurate and average attenuation settings. This deviation decrease as the input power level increases. Figures 6,7 and 8 show the results for $1,1.5$, and $2 \mathrm{GHz}$.

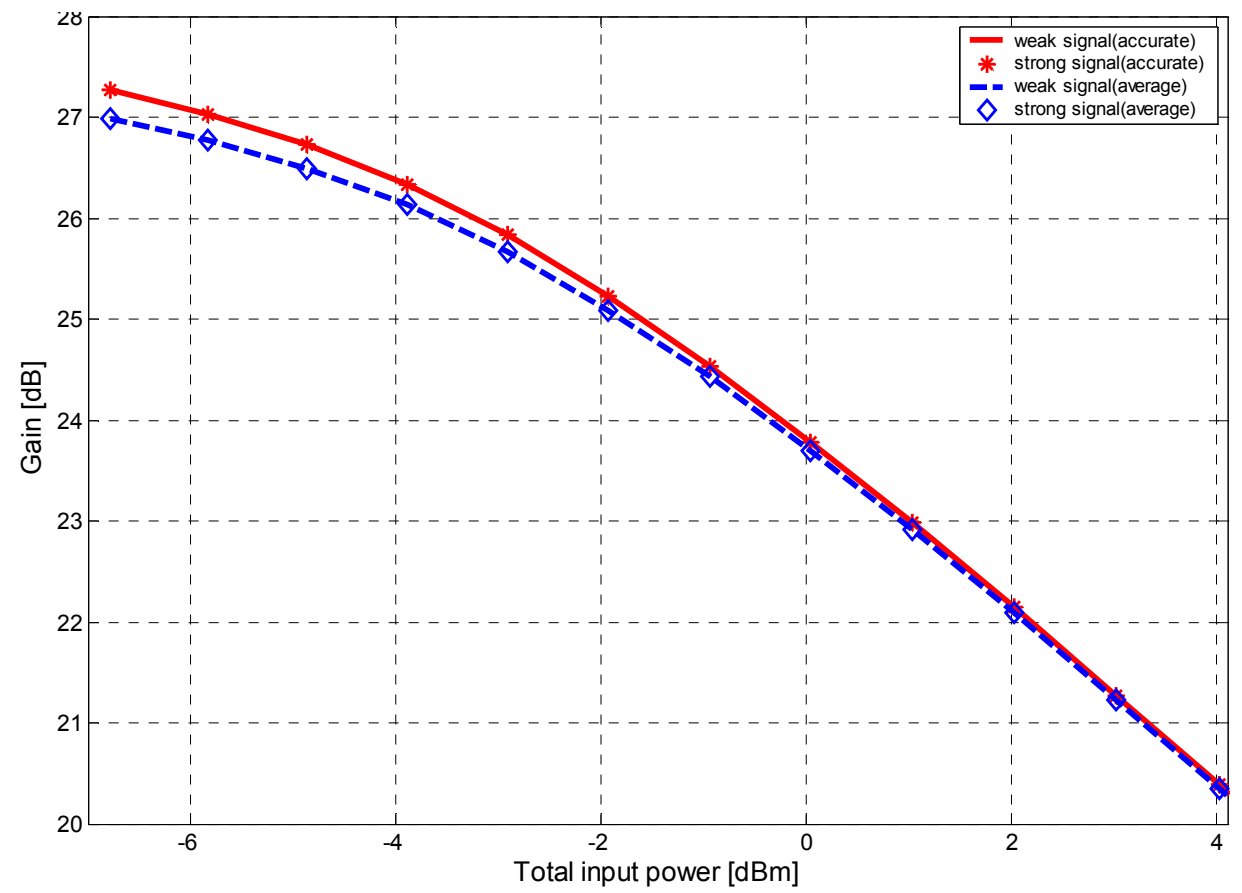

Figure 6: Comparison between the system correction at $1 \mathrm{GHz}$, using accurate and average attenuation settings

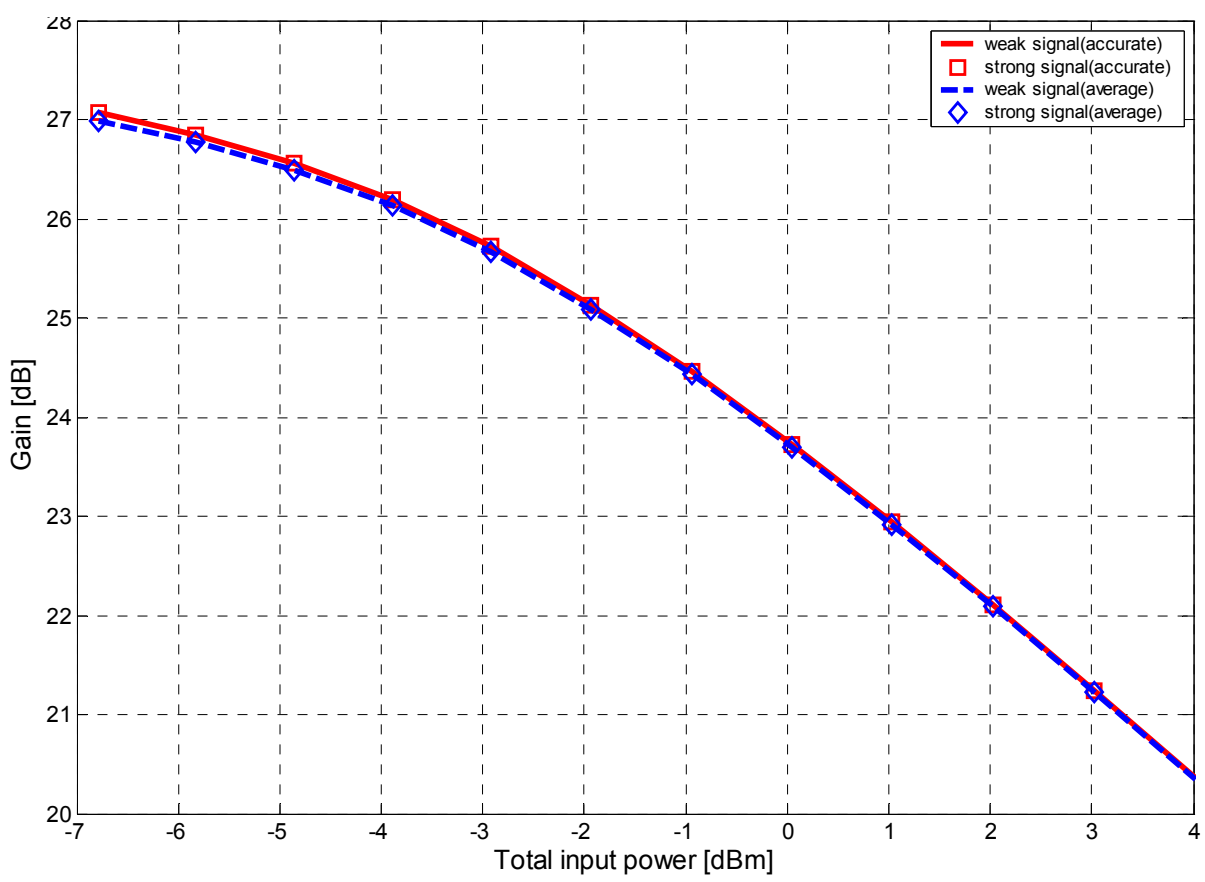

Figure 7: Comparison between the system correction at $1.5 \mathrm{GHz}$, using accurate and average attenuation settings 


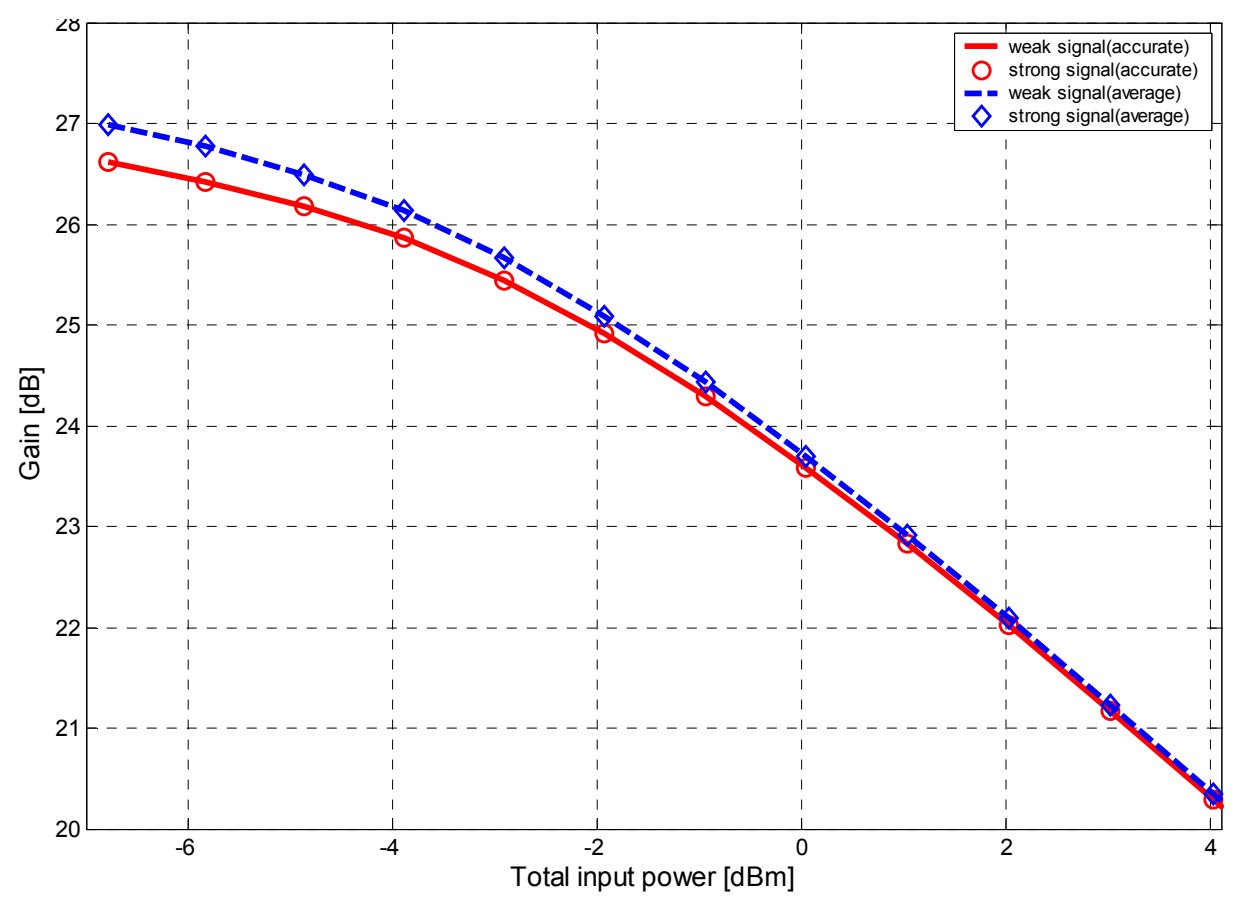

Figure 8: Comparison between the system correction at $2 \mathrm{GHz}$, using accurate and average attenuation settings

\section{CONCLUSION}

1. The concept of gain capture as a criterion for amplifier linearity evaluation is introduced.

2. It is possible to correct the gain capture effect at different levels of input signals using classical Feed-Forward linearization technique, by proper setting of system attenuators and delay lines.

3. The attenuation settings used to minimize $\mathrm{IMD}_{3}$ in the equal two-tone model for a certain total input power can completely correct the gain capture effect for the same total input power.

4. It is possible to find a set of attenuator settings to correct the gain capture effect on a certain frequency band without the need to measure signal frequencies.

\section{REFERENCES}

[1] Joel Vuolevi, Timo Rahkonen, Distortion In RF Power Amplifiers, Artech House, Norwood MA, 2003

[2] Kennington, P., High-Linearity RF Amplifier Design, Norwood, Ma, Artech House, 2000.

[3] F. H. Raab, P. Asbeck, S. Cripps, P. B. Kennington, Z. B. Popovic, N. Pothycarry, J. F. Sevic, and N. O. Sokal, "Power amplifiers and transmitters for RF and Microwave," IEEE Trans. Microwave Theory Tech., vol. 50, no. 3, pp. 814-826,Mar. 2002.

[4] Cripps, Steve C., Advanced Techniques in RF Power Amplifier Design, Artech House, Norwood MA, 2002.

[5] J. K. Cavers, "Adaptation Behavior of a Feedforward Amplifier Linearizer," 
IEEE Trans. Vehicular Technology., vol. 44, no. 1, pp. 31-40, Feb. 1995.

[6] S. J. Grant, J. K. Cavers, "A DSP controlled adaptive Feedforward amplifier linearizer," in Proc. IEEE Int. Conf. Universal Personal Communication, Cambridge, MA, pp.7a8-792, Sep. 1996.

[7] H. Seidel, H. R. Beurrier, and A. N. Friedman, "Error Controlled High Power Linear Amplifiers at VHF," The Bell System Technical J., vol. 47, pp. 651722, May/June 1968.

[8] Dong Jing, and Wing Shing Chan, "New Linearization Method Using Interstage Second Harmonic Enhancement", IEEE Microwave and Guided Wave Letters, Vol 8, no 11, November 1998

[9] Ying Zhang and Payam Heydari, "A Novel linearization technique for Linear/Pseudo linear CMOS power Amplifiers"

[10] K. Konstantinou, P. Gardner and D. K. Paul, "Optimization method for Feedforward linearization of power amplifiers", Electronics Letters 2nd September 1993 Vol. 29, No .18.

[11] Sang-Gee Kang, Kyoo Lee and Ki-Suk Yoo, "Analysis and Design of Feedforward Power Amplifier", IEEE Trans. Microwave Theory Tech., vol. 50, no. 3, pp. 814-826,Mar. 2002. 